\title{
The Role of Dendritic Cells and Immunotherapy in Allergic Fungal Rhinosinusitis
}

\author{
Kenneth D. Rodriguez $\cdot$ Eric W. Wang
}

Published online: 15 March 2013

(C) Springer Science+Business Media New York 2013

\begin{abstract}
This review focuses on dendritic cells and immunotherapy as they relate to allergic fungal rhinosinusitis (AFRS). A comprehensive review using PubMed was performed and articles were reviewed and selected for inclusion based on applicability to our topic of interest and quality of data. Many questions remain unanswered in regards to the management and etiology of AFRS. Much of our current care is driven by small studies and global extrapolation is not possible. Dendritic cells represent an intriguing option for a target cell in the abnormal immune response that is seen with AFRS. Immunotherapy may also play a prominent role in the adjuvant therapy for AFRS but strong data is lacking. AFRS is a challenge to treat and close surveillance as well as consideration of the patient's symptoms, both separate from and combined with clinical or radiographic findings, may be the most valuable medicine we have at present.
\end{abstract}

Keywords Allergic fungal rhinosinusitis .

Dendritic cells $\cdot$ Immunotherapy

\footnotetext{
K. D. Rodriguez

Department of Otolaryngology-Head and Neck Surgery, University of North Carolina, Campus Box 7070, Chapel Hill, NC 27599, USA

e-mail: kenneth_rodriguez@med.unc.edu

E. W. Wang $(\bowtie)$

Department of Otolaryngology, University of Pittsburgh School of Medicine, 200 Lothrop Street, Pittsburgh, PA 15213, USA e-mail: wangew@upmc.edu

E. W. Wang

Eye \& Ear Institute, Suite 500, Pittsburgh, PA 15213, USA
}

\section{Introduction}

Allergic fungal rhinosinusitis (AFRS) is a subtype of chronic rhinosinusitis (CRS) and has traditionally been considered the most difficult grouping of sinusitis to treat secondary to its recalcitrant nature. Little is known about the pathophysiology of AFRS. The epidemiologic data on AFRS is scant with its prevalence between 7-9\% of patients with CRS who undergo sinus surgery in the United States $[1,2]$. Regardless of its actual number, AFRS represents a small percentage of total surgical sinus disease but often require extensive and time consuming surgical procedures to remove the eosinophilic mucin that characterize the disease. A geographic pattern of distribution focused in the southern United States and the Mississippi Basin is observed [3]. The patients are typically young adults with lower socioeconomic backgrounds and tend to be African American [4]. Interestingly, AFRS patients tend to be less bothered by their symptoms resulting in delayed presentations and recurrences. This may in part also contribute to the significant distortion of the bony paranasal sinus anatomy frequently seen in AFRS and staging systems have been created to classify this further [5]. The pathophysiology of AFRS remains unclear. It is suspected that an unidentified trigger leads to an abnormal immune response. Therefore, removing the disease burden eliciting the immune response with surgery and multimodality treatment aimed at immunomodulation are the cornerstones for disease control. It has been hypothesized that immunotherapy (IT) could alleviate inflammation through similar mechanisms to other more commonly treated allergens. It is clear that a Type I hypersensitivity to fungal elements is present in AFRS, yet this does not imply that fungi are the causative agent responsible for the disease. Furthermore, surgical elimination or antifungal drug therapy 
aimed at complete removal of all fungus are not sustainable and, thus, does not necessarily guarantee disease resolution. Studies have shown benefit with IT but its role remains unclear as a foundation of disease treatment.

The exact immunologic dysfunction responsible for AFRS is under investigation. Dendritic cells have garnered recent study because they play a significant role in a number of human respiratory diseases including allergic rhinitis and asthma [6,7]. Dendritic cells are primary antigen-presenting cells involved in interactions with $\mathrm{T}$ cells leading to the proliferation of either T helper 1 (Th1) or T helper 2 (Th2) cell types [8]. The CRS without NP displays elevated levels of Th1 and Th2 cytokines while CRS with NP is more Th2 driven [9]. The AFRS also displays a Th2 skewed phenotype [10]. If a fundamental understanding of the interplay between dendritic cells and the subsequent immune response could be understood in AFRS, it may allow for improved disease control.

\section{Pathophysiology of Disease}

Allergic fungal rhinosinusitis was first recognized by Millar et al. [11] who reported histopathologic similarities between materials obtained from the maxillary sinuses and pathologically diagnosed specimens of allergic bronchopulmonary aspergillosis (ABPA). In 1994, Bent and Kuhn [12] described five criteria for the diagnosis of AFRS: 1. type 1 hypersensitivity by history, skin testing, or serology, 2. nasal polyposis, 3. characteristic CT findings of heterogeneous areas of signal intensity within the sinus cavities, 4. eosinophilic mucous, and 5. positive fungal stain of mucin or from the tissue removed during surgery without fungal invasion. In addition there were six associated criteria: asthma, unilateral predominance, radiographic bone erosion, fungal culture, Charcot-Leyden crystals, and serum eosinophilia.

There remains significant controversy regarding the diagnostic criteria which is driven by differences in philosophy on the role of fungus in the inflammatory response. Kuhn and Swain [13] do note that AFRS has a prolonged time course with possibly years transpiring between disease onset and criteria fulfillment. In addition, the immunological changes may be located within the mucosa of the sinuses and not be a systemic finding. In other words, total serum IgE may not reflect local IgE production. Addressing that point, Chang and Fang [14] demonstrated that $85.7 \%$ of patients with AFRS had tissue IgE specific to Aspergillus. None of their patients had positive serum IgE response to Aspergillus. It may be possible they failed to detect other kinds of fungal IgE due to their limited species testing.

In addition, the role of fungus as the causative agent in AFRS has been questioned. A study in 210 patients with
CRS (with or without polyps) demonstrated positive fungal cultures in $96 \%(202 / 210)$ [15]. They also noted that IgE mediated hypersensitivity to fungal allergens in their AFRS patients was $42 \%$ for skin testing and $30 \%$ for RAST. They also found that fewer than $33 \%$ of patients diagnosed with AFRS had elevated IgE levels. This study raised the question that if $96 \%$ of CRS patients have positive fungal cultures, as well as $100 \%$ of their 14 healthy controls, why do some people develop a profound eosinophilic response? The answer may be at the cellular level.

It has been proposed that ARFS could be driven by $\mathrm{T}$ cell superantigen production and an excellent review of that literature was written by Schubert [16]. Supporting that theory was work done by Ferguson et al. [17] where previously diagnosed eosinophilic chronic rhinosinusitis (ECRS) patients had their pathology re-reviewed in a prospective fashion ( 55 specimens from 34 patients). Their results demonstrated that bacteria were present in $62 \%$ of their specimens and fungus in $40 \%$. The $77 \%$ of fungal colonization was associated with bacteria. They concluded that this could potentially support bacteria as the source of superantigen.

Further complicating our understanding of AFRS is whether it represents a distinct entity or is part of a spectrum of eosinophilic disease. In 2000 a study was published that clarified this ambiguity [18]. A literature review was performed of 431 AFRS patients and 69 patients with eosinophilic mucin rhinosinusitis (EMRS). Among many notable findings, AFS patients compared to EMRS patients were less likely to have asthma (41 vs $93 \%$ ), be aspirin sensitive (13 vs $54 \%$ ), and were more likely to have allergic rhinitis (84 vs $63 \%$ ). Total IgE was significantly more elevated in AFS patients.

To further determine the inflammatory response of noninvasive fungal sinusitis, Carney et al. [10] performed infundibular mucosal tissue harvested from patients with AFRS, non-allergic eosinophilic fungal sinusitis (NEFS), CRS, and normal controls. They determined that mast cell, eosinophil, and IgE cell numbers were significantly raised in patients with AFRS, NEFS, and CRS when compared with controls. There was no significant difference between cell numbers in patients with AFRS and NEFS. They determined that AFRS has an immunologic response indicative of a Th2 cascade.

The MUSC group has explored the role of dendritic cells in the immune response of AFRS. Dendritic cells are professional antigen presenting cells which are capable of inciting a Th1 or Th2 response. To determine if there was a link between local dendritic cells population and CRS with NP, CRS without NP, and AFRS, Ayers et al. [19] performed immunohistochemical staining for dendritic cells within the mucosa of each CRS subtype and found that AFRS and CRS with NP had increased numbers of dendritic cells as compared to controls. No difference in the 
number of dendritic cells or levels of dendritic cell chemoattractants was seen between controls and CRS without NP or AFRS and CRS with NP. They postulate that this increase in dendritic cell number may represent a possible mechanism why the Th2 profile is noted in both CRS with NP and AFRS.

Similarly the relationship of dendritic cells and vitamin $\mathrm{D}_{3}\left(\mathrm{VD}_{3}\right)$ is currently under investigation. The $\mathrm{VD}_{3}$ can block monocyte to dendritic cell differentiation and maturation, thereby diminishing dendritic cell's ability to subsequently stimulate $\mathrm{T}$ cell $\mathrm{Th} 1 / \mathrm{Th} 2$ differentiation [20]. Additionally $\mathrm{VD}_{3}$ has characteristics that are molecularly similar to corticosteroids, a frequently utilized treatment strategy in AFRS. The AFRS and $\mathrm{VD}_{3}$ deficiency is more prevalent in African Americans who are noted to have more severe bone erosion [4, 21]. A retrospective analysis of $\mathrm{VD}_{3}$ levels in patients with AFRS CRS with NP, CRS without polyps and controls demonstrated that both CRS with NP and AFRS were associated with insufficient levels of $\mathrm{VD}_{3}$ [22]. The $\mathrm{VD}_{3}$ levels were inversely correlated with circulating numbers of mature DCs, DC regulatory factors, and bone erosion. In contrast, CRS without nasal polyps displayed increased circulating macrophages. The $\mathrm{VD}_{3}$ deficiency was associated with more severe bone erosion. A similar study was performed in the pediatric population [23]. Examining pediatric patients with AFRS, CRS with NP, CRS without NP and controls, no difference in mean $\mathrm{VD}_{3}$ levels between control and CRS without NP was seen, whereas mean CRS with NP and AFRS levels were both low. The relationship between low $\mathrm{VD}_{3}$ levels and decreased levels of dendritic cells in the sinus mucosa remained consistent with the adult population.

While the current studies on dendritic cells and $\mathrm{VD}_{3}$ in AFRS and CRS with NP are suggestive, they remain observational and correlative at present. Correction of the $\mathrm{VD}_{3}$ levels have yet to be shown to reduce the inflammation associated with AFRS.

\section{Treatment}

Allergic fungal sinusitis is a disease for which optimal treatment remains unclear. Treatment has traditionally consisted of both surgical and medical therapy. Although the need for complete surgical removal is unlikely to change in the near future, the interplay between surgery and pre or post-operative medical therapy is likely to advance greatly as more studies uncover the mechanism behind this disease's tenacity. The surgical goal should be complete removal of all eosinophilic mucin and re-establishment of drainage pathways. As with other inflammatory sinus surgery, mucosal preservation should be the ultimate goal. Failure to remove all disease will lead to poor outcomes
[24]. Assuming complete surgical removal of disease, postoperative medical care and surveillance is critical.

\section{Corticosteroids}

Most studies have focused on oral corticosteroids to decrease disease recurrence in AFRS. It has been suggested that preoperative steroids with oral antibiotics may decrease polyp burden and treat post-obstructive bacterial rhinosinusitis $[25,26]$. However, this is certainly not uniformly employed and there is not sufficient evidence to support this recommendation. Ikram et al. [27] evaluated 63 patients with AFRS who underwent endoscopic sinus surgery with $(N=33)$ or without postoperative steroid therapy which included oral and topical therapies $(N=30)$. Their minimum follow up was 2 years and their results demonstrated that $50.0 \%(15 / 30)$ of the no-steroid group and $15.2 \%$ $(5 / 33)$ of the steroid group had recurrent symptoms. Rupa et al. [28] performed a prospective, randomized, placebo controlled trial of post-operative oral steroid use in AFRS. Twenty-four patients diagnosed with AFRS underwent sinus surgery and were randomized to receive either oral steroid $(N=12)$ or placebo $(N=12)$ after surgery. All patients were also administered itraconazole and steroid nasal spray in the postoperative period. Their results demonstrated statistically significant subjective and objective improvement of patients with AFRS treated with steroids and that steroids are also effective in preventing early recurrence.

Unfortunately oral steroids are not a panacea. An excellent review on this topic can be found through UpToDate [29•]. Generally speaking, the lowest dose and shortest duration of oral steroids are best to avoid complications from those medications. Benefits must be weighed against patient symptoms and their tolerance for side effects. In contrast, topical corticosteroids are generally accepted as a standard therapy in the postoperative treatment of AFRS when the sinuses are widely patent allowing for penetration of the spray to the exposed mucosa. The side effect profile is minimal. Unfortunately, there are no studies demonstrating the benefit of topical nasal steroids in AFRS and the data is extrapolated from studies of CRS with NP [30].

The goal of IT is to decrease symptoms associated with allergic rhinitis. This is accomplished through complicated mechanisms that are not completely elucidated. The IT is accompanied by increases in allergic-specific IgG (particularly IgG4) which block IgE dependent histamine release and also IgE mediated presentation to T-cells [31]. In addition, it modifies mucosal Th2 responses in favor of Th1 [31]. Further specifics in regards to cytokine production are beyond the scope of this text but are active topics of current research.

All patients are constantly being exposed to aerosolized fungal allergens. Noble et al. [32] found that fungi recovered 
from air samples from patients' residences included the same species isolated from the mucin of its inhabitants meaning there is essentially constant exposure to antigen. In this context IT would be a reasonable extension of care to abate and regulate the immune and inflammatory response.

Mabry and colleagues [33-37] have the largest experience in the current literature with IT and AFRS. Beginning in 1994, a study group of AFRS were initiated on fungal IT following surgical intervention and prospectively followed. Initially theoretical concerns for safety limited their cohort to nine patients on fungal IT alone with the introduction of nonfungal antigens after repeat RAST testing at 6 months [33]. After demonstrating safety, all antigen IT was started at the onset. After 1 year (4-12 months range with 8.6 month average) they noted decreased crusting and allergic mucin and decreased need for topical and systemic steroids. Most importantly they noted no deleterious effects from the IT. The study was expanded to 2- and 3-year follow-up [34, 35]. At the 2-year follow they had a total of ten patients of which two underwent revision ESS. At 3 years they had 11 patients who had at least 1 year of IT with a mean of 28 months. Again they note positive results in regards to decrease need for oral steroids as well as decreased recurrence rates of AFRS. The authors admit that lack of a placebo group is a shortcoming of the studies as well as differences in use of topical steroids but ultimately this study did demonstrate safety of IT with fungal antigens in this group of patients with AFRS.

The group later published on 22 AFRS patients with similar treatment only differing in whether they received IT [36]. After a mean of 33 months of treatment their results demonstrated significant improvement in patient outcome both subjectively and objectively for those undergoing IT. The IT was also shown to reduce reliance on systemic and topical nasal steroid therapy to control disease. This study reinforced their previous positive findings in regards to IT.

In 2000, they published on outcomes after discontinuing IT for AFRS [37]. Eight patients who had received at least 3 years (range 36-52 months-mean 40 months) of IT who had been off IT for 7-17 months (mean 13 months) were evaluated. They noted that none of the patients had evidence of recurrent disease.

Greenhaw et al. [38] later performed a study with 14 patients with AFRS compared to 14 controls to determine the safety of high-dose subcutaneous fungal IT in patients with allergic fungal sinusitis. Their data demonstrated that subcutaneous high dose fungal IT in patients with AFRS is unlikely to cause adverse reactions other than those occurring with pollen IT.

A concise review of IT for AFRS was written by Hall and DeShazo [39•]. They note that IT should be considered as part of treatment for AFRS, but given the small number of patients in the current body of literature, controlled studies are critical to clarify the role of IT.

\section{Outcomes}

Recurrence rates of AFRS are highly variable. Multiple factors may play a role including the variability in surgical technique which often is not addressed and post-operative use of oral steroids, topical steroids, and/or IT. In addition, patients may often have endoscopic evidence of mucosal relapses without significant symptoms. As part of routine surveillance, many physicians use the mucosal staging system developed by Kuferberg et al. [40] which classifies mucosa as; stage 0: no mucosal edema or allergic mucin, stage I: mucosal edema with or without allergic mucin, stage II: polypoid edema with or without allergic mucin, and stage III: sinus polyps with allergic mucin.

The longest follow up to date was conducted by Marple et al. [41]. They reviewed 17 patients with follow up ranging from $46-138$ months (mean 82 months) at UT Southwestern. All patients in their study met the AFRS criteria set forth by Kuhn. They note $76 \%$ of patients with normal mucosa or mild edema (stage 0-1) with $6 \%$ demonstrating recurrence at their latest follow up. Their patients received systemic steroids, nasal steroids, IT $(N=10)$ and antibiotics as needed. There was no difference between the two groups in regards to outcomes with and without IT. Average number of operations for their cohort was 2.

Ultimately several conclusions can be drawn. At centers that treat AFRS frequently, clinical disease control can be achieved in about $75 \%$ of patients with long term follow up. Unfortunately, extrapolation beyond this study is limited. This disease is seen by numerous physicians across the country and even the diagnostic criteria remain heterogeneous. Only until pooled data can be obtained from multiple centers with standardized diagnosis and treatment algorithms can the true recurrence rate be known. In addition, how is success defined: radiographic, endoscopic evaluation or quality of life? These three endpoint measures may yield entirely different findings. If a patient's chief complaint on presentation was hyposmia or nasal obstruction and those symptoms were alleviated by surgery are they a treatment failure if they have higher stage mucosal inflammation? Ultimately, complete resolution of symptoms and a normal appearing endo-nasal exam may be the end point. However a balance between the benefits and side effects of therapy must be considered and thought must be given about how much adjuvant therapy patients need without significant symptoms.

\section{Conclusions}

Many questions remain unanswered with AFRS. What are the specific immunologic differences that drive the disease 
process and are they unique from other forms of sinusitis such as eosinophilic rhinosinusitis? What is the role for fungus in this disease? How does unilateral disease occur? While the current standard of endoscopic sinus surgery for clearance of the eosinophilic mucin followed by control of inflammation can be efficacious, further study in the pathophysiology and subsequent treatment of AFRS is needed. Dendritic cells may represent one target cell. The IT may also play a prominent role in the adjuvant therapy for AFRS. No evidence based recommendation can be provided given the small studies available at present. Nevertheless, AFRS is a challenge to treat and close surveillance as well as consideration of the patient's symptoms, both separate from and combined with clinical or radiographic findings, may be the most valuable medicine we have.

Disclosure No potential conflicts of interest relevant to this article were reported.

\section{References}

Papers of particular interest, have been highlighted as:

- Of importance

1. Schwietz LA, Gourley DS. Allergic fungal sinusitis. Allergy Proc. 1992;13(1):3-6.

2. Granville L, Chirala M, Cernoch P, et al. Fungal sinusitis: histologic spectrum and correlation with culture. Hum Pathol. 2004;35(4):474-81.

3. Ferguson BJ, Barnes L, Bernstein JM, et al. Geographic variation in allergic fungal rhinosinusitis. Otolaryngol Clin $\mathrm{N}$ Am. 2000;33(2):441-9.

4. Wise SK, Ghegan MD, Gorham E, Schlosser RJ. Socioeconomic factors in the diagnosis of allergic fungal rhinosinusitis. Otolaryngol Head Neck Surg. 2008;138:38-42.

5. Wise SK, Rogers GA, Ghegan MD, et al. Radiologic staging system for allergic fungal rhinosinusitis (AFRS). Otolaryngol Head Neck Surg. 2009;140(5):735-40.

6. Lipscomb MF, Masten BJ. Dendritic cells: immune regulators in health and disease. Physiol Rev. 2002;82:97-130.

7. Hartmann E, Graefe H, Hopert A, et al. Analysis of plasmacytoid and myeloid dendritic cells in nasal epithelium. Clin Vaccine Immunol. 2006;13:1278-86.

8. Bharadwaj AS, Bewtra AK, Agrawal DK. Dendritic cells in allergic airway inflammation. Can J Physiol Pharmacol. 2007;85:686-99.

9. Schleimer RP, Kato A, Peters A, et al. Epithelium, inflammation, and immunity in the upper airways of humans: studies in chronic rhinosinusitis. Proc Am Thorac Soc. 2009;6:288-94.

10. Carney AS, Tan LW, Adams D, et al. Th2 immunological inflammation in allergic fungal sinusitis, nonallergic eosinophilic fungal sinusitis, and chronic rhinosinusitis. Am J Rhinol. 2006;20(2):145-9.

11. Millar JW, Johnston A, Lamb D. Allergic aspergillosis of the maxillary sinuses (abstract). Thorax. 1981;36:710.

12. Bent JP, Kuhn FA. Diagnosis of allergic fungal sinusitis. Otolaryngol Head Neck Surg. 1994;111:580-8.
13. Kuhn FA, Swain R Jr. Allergic fungal sinusitis: diagnosis and treatment. Curr Opin Otolaryngol Head Neck Surg. 2003;11(1): $1-5$.

14. Chang YT, Fang SY. Tissue-specific immunoglobulin E in maxillary sinus mucosa of allergic fungal sinusitis. Rhinology. 2008;46(3):226-30.

15. Ponikau JU, Sherris DA, Kern EB, et al. The diagnosis and incidence of allergic fungal sinusitis. Mayo Clin Proc. 1999;74(9): 877-84.

16. Schubert MS. A superantigen hypothesis for the pathogenesis of chronic hypertrophic rhinosinusitis, allergic fungal sinusitis, and related disorders. Ann Allergy Asthma Immunol. 2001;87(3): $181-8$.

17. Ferguson BJ, Seethala R, Wood WA. Eosinophilic bacterial chronic rhinosinusitis. Laryngoscope. 2007;117(11):2036-40.

18. Ferguson BJ. Eosinophilic mucin rhinosinusitis: a distinct clinicopathological entity. Laryngoscope. 2000;110(5 Pt 1):799-813.

19. Ayers CM, Schlosser RJ, O'Connell BP, et al. Increased presence of dendritic cells and dendritic cell chemokines in the sinus mucosa of chronic rhinosinusitis with nasal polyps and allergic fungal rhinosinusitis. Int Forum Allergy Rhinol. 2011;1(4):296-302.

20. Hackstein H, Thomson AW. Dendritic cells: emerging pharmacological targets of immunosuppressive drugs. Nat Rev Immunol. 2004;4:24-34.

21. Thomas MK, Demay MB. Vitamin D deficiency and disorders of vitamin D metabolism. Endocrinol Metab Clin N Am. 2000;29(3): 611-27.

22. Mulligan JK, Bleier BS, O'Connell B, et al. Vitamin D3 correlates inversely with systemic dendritic cell numbers and bone erosion in chronic rhinosinusitis with nasal polyps and allergic fungal rhinosinusitis. Clin Exp Immunol. 2011;164(3):312-20.

23. Mulligan JK, White DR, Wang EW, et al. Vitamin D3 deficiency increases sinus mucosa dendritic cells in pediatric chronic rhinosinusitis with nasal polyps. Otolaryngol Head Neck Surg. 2012;147(4):773-81.

24. Marple BF, Mabry RL. Allergic fungal sinusitis: learning from our failures. Am J Rhinol. 2000;14(4):223-6.

25. Marple BF. Allergic fungal rhinosinusitis: current theories and management strategies. Laryngoscope. 2001;111(6):1006-19.

26. Marple BF, Mabry RL. Comprehensive management of allergic fungal sinusitis. Am J Rhinol. 1998;12:263-8.

27. Ikram M, Abbas A, Suhail A, et al. Management of allergic fungal sinusitis with postoperative oral and nasal steroids: a controlled study. Ear Nose Throat J. 2009;88(4):E8-11.

28. Rupa V, Jacob M, Mathews MS, Seshadri MS. A prospective, randomised, placebo-controlled trial of postoperative oral steroid in allergic fungal sinusitis. Eur Arch Otorhinolaryngol. 2010;267(2): $233-8$.

29. - Saag KG, Furst DE. Major side effects of systemic glucocorticoids. www.uptodate.com. Excellent review of the side effects of oral steroids.

30. Joe SA, Thambi R, Huang J. A systematic review of the use of intranasal steroids in the treatment of chronic rhinosinusitis. Otolaryngol Head Neck Surg. 2008;139(3):340-7.

31. Till SJ, Francis JN, Nouri-Aria K, Durham SR. Mechanisms of Immunotherapy. J Allergy Clin Immunol. 2004;113:1025-34.

32. Noble JA, Crow SA, Ahearn DG, Kuhn FA. Allergic fungal sinusitis in the southeastern USA: involvement of a new agent Epicoccum nigrum Ehrenb. ex Schlecht. 1824. J Med Vet Mycol. 1997;35(6):405-9.

33. Mabry RL, Manning MC, Mabry CS. Immunotherapy in the treatment of allergic fungal sinusitis. Otorhinolaryngol Head Neck Surg. 1997;116:31-5.

34. Mabry RL, Mabry CS. Immunotherapy for allergic fungal sinusitis: the second year. Otorhinolaryngol Head Neck Surg. 1997;117:367-71. 
35. Mabry RL, Marple BF, Folker RJ, Mabry CS. Immunotherapy for allergic fungal sinusitis: three year's experience. Otorhinolaryngol Head Neck Surg. 1998;119:648-51.

36. Folker RJ, Marple BF, Mabry RL, Mabry CS. Treatment of allergic fungal sinusitis: a comparison trial of postoperative immunotherapy with specific fungal antigens. Laryngoscope. 1998;108(11 Pt 1):1623-7.

37. Mabry RL, Marple BF, Mabry CS. Outcomes after discontinuing immunotherapy for allergic fungal sinusitis. Otolaryngol Head Neck Surg. 2000;122(1):104-6.

38. Greenhaw B, de Shazo RD, Arnold J, Wright L. Fungal immunotherapy in patients with allergic fungal sinusitis. Ann Allergy Asthma Immunol. 2011;107(5):432-6.
39. - Hall AG, Deshazo RD. Immunotherapy for allergic fungal sinusitis. Curr Opin Allergy Clin Immunol. 2012;12(6):629-634. Represents a comprehensive review of IT for AFRS.

40. Kupferberg SB, Bent JP 3rd, Kuhn FA. Prognosis for allergic fungal sinusitis. Otolaryngol Head Neck Surg. 1997;117(1):35-41.

41. Marple B, Newcomer M, Schwade N, Mabry R. Natural history of allergic fungal rhinosinusitis: a 4- to 10-year follow-up. Otolaryngol Head Neck Surg. 2002;127(5):361-6. 